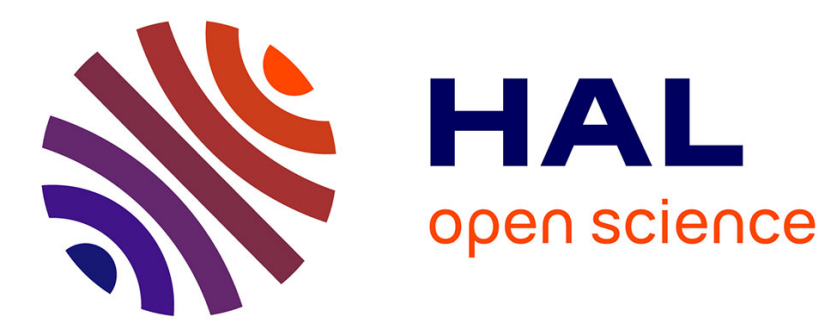

\title{
Atomic-Scale Modelling of Electrochemical Interfaces through Constant Fermi Level Molecular Dynamics
}

Assil Bouzid, Alfredo Pasquarello

\section{To cite this version:}

Assil Bouzid, Alfredo Pasquarello (Dir.). Atomic-Scale Modelling of Electrochemical Interfaces through Constant Fermi Level Molecular Dynamics. Wiley, 1, 2021, 10.1002/9781119605652.ch7 . hal-03428392

\section{HAL Id: hal-03428392 \\ https://hal.science/hal-03428392}

Submitted on 15 Nov 2021

HAL is a multi-disciplinary open access archive for the deposit and dissemination of scientific research documents, whether they are published or not. The documents may come from teaching and research institutions in France or abroad, or from public or private research centers.
L'archive ouverte pluridisciplinaire HAL, est destinée au dépôt et à la diffusion de documents scientifiques de niveau recherche, publiés ou non, émanant des établissements d'enseignement et de recherche français ou étrangers, des laboratoires publics ou privés. 


\title{
Atomic-Scale Modelling of Electrochemical Interfaces through Constant Fermi Level Molecular Dynamics
}

\author{
Assil Bouzid and Alfredo Pasquarello
}

\begin{abstract}
Electrochemical reactions involving electron/proton transfer occur in several technologically relevant environments, such as solar cells, energy conversion, and storage devices. In these systems, the electron/proton transfer is governed by the applied bias potential and leads to the occurrence of complex electrochemical reactions. While experiments provide access to most of the macroscopic electrochemical properties of these reactions, they are unable to describe the electrochemical processes occurring at the atomic scale. At this level, modelling electrochemical processes either in solution or at metal interfaces is of paramount importance towards a full control and understanding of reaction mechanisms occurring under applied bias potential. Several techniques have been developed to study systems under bias potential in combination with ab initio molecular dynamics and advanced electronic structure techniques. Among them, constant Fermi level ab initio molecular dynamics (CFL-MD) is emerging as a promising and efficient scheme. In this Chapter, we focus on the constant Fermi level molecular dynamics technique and present some applications to electrochemistry. In the methods section, we provide a detailed review of the constant Fermi level ab initio molecular dynamics technique. Then, we illustrate the performance of CFL-MD in determining redox levels of half reactions in solution. In particular, we show that redox half-reactions can be driven by varying the Fermi level within the electronic gap of liquid water, thereby revealing reaction mechanisms without any prior knowledge of the reaction products. On the basis of Janak's theorem, we derive a scheme for determining redox potentials from the evolution of single-particle energy levels upon charging (or discharging) the system. For the considered redox couples, the calculated redox levels are found to agree closely with those obtained via the thermodynamic integration method. The agreement with
\end{abstract}

Assil Bouzid,

Institut de Recherche sur les Céramiques Centre Européen de la Céramique, 12 Rue Atlantis, 87068

Limoges, e-mail: assil.bouzid@unilim.fr

Alfredo Pasquarello,

Chaire de Simulation à l'Echelle Atomique (CSEA), Ecole Polytechnique Fédérale de Lausanne (EPFL), CH-1015 Lausanne, Switzerland, e-mail: alfredo.pasquarello@epfl.ch 
experiment is consistent with the accuracy of the adopted level of theory. In the following section, we then consider the case of metal-water interfaces with a particular focus on the spontaneous occurrence of chemical reactions. Specifically, we show how chemical reactions (e.g. the Volmer reaction) can be induced using the CFLMD technique and discuss the subsequent reaction pathway. In addition to chemical reactions, the CFL-MD offers the possibility of studying electrochemical properties of metal/water interfaces under applied bias potential. However, this requires a proper alignment of the electrode potential to the standard hydrogen electrode (SHE) level. To achieve this, we introduce an alignment procedure, which includes a correction based on classical electrostatics to account for the charge compensation in the simulation cell. As a consequence of this alignment, we extract macroscopic properties of the $\mathrm{Pt}(111) /$ water interface under variable bias potential. Our scheme yields a potential of zero charge of $0.22 \mathrm{eV}$ relative to the SHE and a double layer capacitance $\mathrm{Cdl}$ of $\sim 19 \mu \mathrm{F} \mathrm{cm} \mathrm{cm}^{-2}$, in good agreement with experimental values. Moreover, we achieve an atomic-scale description of the structural reorganization of the water bilayer at the Pt surface as a function of the applied voltage.

\section{Introduction}

Electrochemical reactions involving electron/proton transfer at metal/water interfaces occur in several technologically relevant environments, such as solar cells, energy conversion, and storage devices. In these systems, the electron/proton transfer is governed by the applied bias potential, which defines the amount of excess charge at the interface. Hence, modelling the metal/water electrochemical interface at constant electrode potential is of paramount importance towards controlling and understanding the electrochemical properties of the interface. In practice, this task is not trivial and several challenges are faced when density functional theory (DFT) based methods are used within periodically repeated cells. First, the control of the electrode potential is still at a pioneering stage and a universal grand canonical method is still not available. Second, the lack of an alignment scheme to simulate systems at well defined electrode potential represents a major issue toward the advancement of this field. In this context, an ideal modelling scheme should, on the one hand, allow one to reference the electrode potential to the standard hydrogen electrode (SHE), and, on the other hand, give access to the reaction mechanisms and the structural properties of the electrical double layer at the interface with the electrode.

Previous modelling schemes studied the metal/water interface through either an implicit $[1,2,3,4]$ or an explicit but static [5, 6] solvent. In the latter case, only few layers were considered. These models were used to access the hydrogen and oxygen evolution reactions. Recently, interface models from first-principles molecular dynamics (MD) with explicit water have given access to the potential of zero charge (pzc) at the $\mathrm{Pt}(111) /$ water interface [7, 8]. A further step consists in modelling the metal/water interface under variable bias potential. In a pioneering work, 
Lozovoi et al. [9] modelled electrified interfaces by allowing the slab to exchange electrons with a reference electrode under constant preset chemical potential. While this approach made possible the simulation of charged slabs like in electrochemical set-ups, it suffered from strong fluctuations of the electron density and electronic structure calculations were difficult to converge $[9,10]$. Subsequently, Otani et al. [11] and Jinnouchi et al. [1] introduced alternative schemes to study the dynamics at the solvent/electrode interface at fixed excess charge $[12,13]$. More recently, Bonnet et al. performed molecular dynamics simulations at constant electrode potential [10], in which the metal/electrolyte interface was simulated in a non-repeated cell. In this modeling scheme, an effective screening medium [11] was adopted to ensure the charge neutrality of the simulation cell. This technique revealed mechanistic aspects of electrochemical processes at metal/water interfaces [14, 12], but the energetics were affected and the band alignment was thus difficult to access.

As far as the band alignment is concerned, the standard hydrogen electrode (SHE) as set by Cheng and Sprik enables a direct comparison of the computed electrochemical energy levels to experiments either in bulk liquid water or at electrode/water interfaces $[15,8]$. This technique is based on a Born-Haber cycle linking the aqueous $\mathrm{H}_{\mathrm{aq}}^{+}$to $\mathrm{H}^{+}(\mathrm{g})$ in the gas phase and the associated energy level is evaluated through the Gibbs free energies of the reaction. Rossmeisl et al. introduced a generalized scheme, in which not only the potential, but also the $\mathrm{pH}$ could be varied $[16,17]$. This method constructs the grand canonical potential by performing several simulations at different $\mathrm{pHs}$ and electrode potentials and uses the experimental SHE to determine the calculated work function of each configuration on an absolute energy scale. While these methods advanced the field and improved our understanding of electrochemical interfaces, none of them provides at the same time a simulation at constant bias potential referenced to the SHE and a description of the mechanistic aspects of electrochemical reactions at the atomic scale.

In this contribution, we first review the methodology behind the constant Fermi level molecular dynamics. We then validate our theoretical framework in the determination of redox levels of aqueous species. Next, we apply the constant Fermi level technique to the study of metal-water interfaces. In particular, we focus on the study of the mechanistic aspects of the Volmer reaction. Furthermore, we extend the technique to reference the applied potential to the SHE in the case of metal-water interface. This procedure allows us to study, at the one hand, the macroscopic properties at the metal-water interface and, on the other hand, the atomic-scale processes at the metal-water interface.

\section{Method}

In this section, we review the dynamical equations for achieving constant Fermilevel simulations, as introduced by Bonnet et al. [10]. We consider a system of particles described by a set of atomic positions $r_{i}$ and a total electronic charge $N_{e}$. To control the Fermi level, this system is connected to an external potentiostat 
at a fixed Fermi level $\bar{\epsilon}_{\mathrm{F}}$ acting like an electron reservoir or a fictitious external electrode. The electronic charge $N_{e}$ is considered as a dynamical variable with inertia $M_{e}$ and the extended system is driven by the grand canonical potential [10]: $\Omega=E^{\text {tot }}\left(r_{i}, N_{e}\right)-N_{e} \bar{\epsilon}_{\mathrm{F}}$, where $E^{\mathrm{tot}}\left(r_{i}, N_{e}\right)$ is the total energy in the absence of the electron reservoir and $N_{e} \bar{\epsilon}_{\mathrm{F}}$ the energy of $N_{e}$ electrons in the reservoir. According to this definition, the forces acting on the atoms and the electronic charges are given by [10]:

$$
F_{i}=-\frac{\partial E^{\mathrm{tot}}\left(r_{i}, N_{e}\right)}{\partial r_{i}} \quad \text { and } \quad F_{e}=-\left(\epsilon_{\mathrm{F}}-\bar{\epsilon}_{\mathrm{F}}\right),
$$

where $\epsilon_{\mathrm{F}}$ is the instantaneous Fermi energy. $F_{e}$ drives electrons into the system when $\epsilon_{\mathrm{F}}<\bar{\epsilon}_{\mathrm{F}}$ and drives electrons into the reservoir when $\epsilon_{\mathrm{F}}>\bar{\epsilon}_{\mathrm{F}}$, so that the instantaneous Fermi energy $\epsilon_{\mathrm{F}}$ averages out to the preset $\bar{\epsilon}_{\mathrm{F}}$. The dynamical equations for the charge evolution then read:

$$
\dot{N}_{e}=\frac{P_{e}}{M_{e}} \quad \text { and } \quad \dot{P}_{e}=F_{e}=-\left(\epsilon_{\mathrm{F}}-\bar{\epsilon}_{\mathrm{F}}\right),
$$

where $P_{e}$ is a fictitious momentum associated to the dynamical variable $N_{e}$.

In this technique, the electronic charge in the system is controlled in an analogous way as the temperature and the pressure in constant-temperature $[18,19,20,21]$ and constant-pressure [22] molecular dynamics, respectively. To control the fluctuations of $N_{e}$ and consequently those of $\epsilon_{\mathrm{F}}$, it is also possible to couple the charge dynamics to a separate thermostat set at the temperature $T_{e}$ [10]. This does not create any significant thermal flow in the system, due to the weak coupling between the atoms and the charges [10].

For a good choice of $M_{e}$ and $T_{e}$, the instantaneous Fermi level of the system is governed by the preset $\bar{\epsilon}_{\mathrm{F}}$. In the case of redox reactions, performing MD at constant Fermi level corresponds to defining the net charge in the system. Hence, by controlling $\bar{\epsilon}_{\mathrm{F}}$, one can drive the reaction from the reactants in a charge state $q$ to the products in a charge state $q^{\prime}$, and get the structural transformations induced by the charge transfer.

\section{CFL-MD in aqueous solution: Determination of redox levels}

We focus on the determination of redox levels of aqueous ions. To this end, we resort to density functional theory (DFT) through the rVV10 functional [23, 24], which is based on a generalized gradient approximation for the exchange-correlation energy and augmented to include nonlocal van der Waals interactions. Within this DFT scheme, the short-range interactions are controlled via an empirical parameter $b$. We adopted the value of $b=9.3$ [25]. The core-valence interactions were described by normconserving pseudopotentials according to the prescription of Troullier and Martins [26]. The wave functions of the valence electrons were expanded on a planewave basis set defined by a kinetic energy cutoff of 80 Ry. The Brillouin zone was 

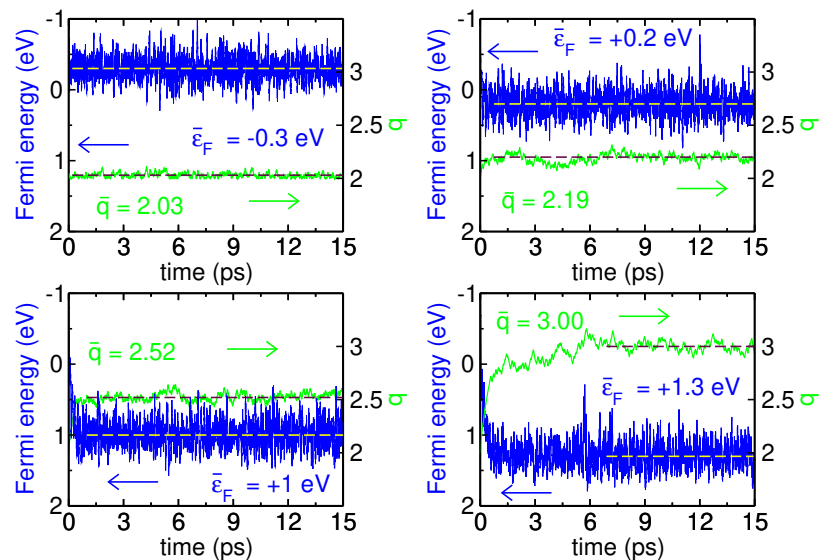

Fig. 1 Time evolution of the instantaneous Fermi level $\epsilon_{\mathrm{F}}$ (blue lines) and total electronic charge $q$ (green lines) in constant Fermi-level molecular dynamics of the aqueous $\mathrm{Fe}^{+2} / \mathrm{Fe}^{+3}$ redox couple. The panels correspond to four different preset values of the Fermi energy $\bar{\epsilon}_{\mathrm{F}}:-0.3,+0.2,+1.0$, and $+1.3 \mathrm{eV}$. The average values after equilibration are given for both $\epsilon_{\mathrm{F}}$ and $\bar{q}$ (dashed lines). The energies are referred to the SHE.

sampled at the $\Gamma$ point. We performed Born-Oppenheimer molecular dynamics using a time step of $\Delta t=0.48$ fs to integrate the equations of motion. We used a velocity rescaling method to set the temperature at $T=350 \mathrm{~K}$. The aqua ion was simulated with 31 water molecules in a cubic supercell with a side of $9.85 \AA$. We used the suite of codes provided in the Quantum-ESPRESSO package [27].

The cell charge neutrality is ensured through a uniform neutralizing background. At each MD step, the electronic charge is first computed via eq 2. Then, the electronic structure is minimized at this fixed charge. Next, the atomic positions and the electronic charge are updated. In this work, the energy levels were referred to the standard hydrogen electrode, for which we took the level calculated in ref [28] at the same level of theory. With this choice of alignment, the conduction band minimum and the valence band maximum of liquid water occur at -2.5 and $1.7 \mathrm{eV}$, respectively. A complete description of the computational setup is provided in ref [29].

We validate our method in the case of the reduction of the $\mathrm{Fe}^{+3}$ aqua ion:

$$
\mathrm{Fe}^{+3}+e^{-} \rightarrow \mathrm{Fe}^{+2}
$$

After 3 ps of equilibration of $\mathrm{Fe}^{+3}$ at $350 \mathrm{~K}$, the last configuration has been taken as the starting point of several runs at different Fermi energies $\bar{\epsilon}_{\mathrm{F}}$, as shown in Figure 1. The choice of the target Fermi energies is guided by the energy level of the lowest unoccupied single-particle state of $\mathrm{Fe}^{+3}$. The occupation number of this state goes from 0 to 1 as the reduction proceeds. In practice, the instantaneous Fermi energy goes within $\sim 1$ ps to the target value $\bar{\epsilon}_{\mathrm{F}}$ by taking on a fractional number of electrons from the potentiostat. After this period, the time averages of the instantaneous Fermi energy are taken over at least $10 \mathrm{ps}$ and are found to agree with the target value 

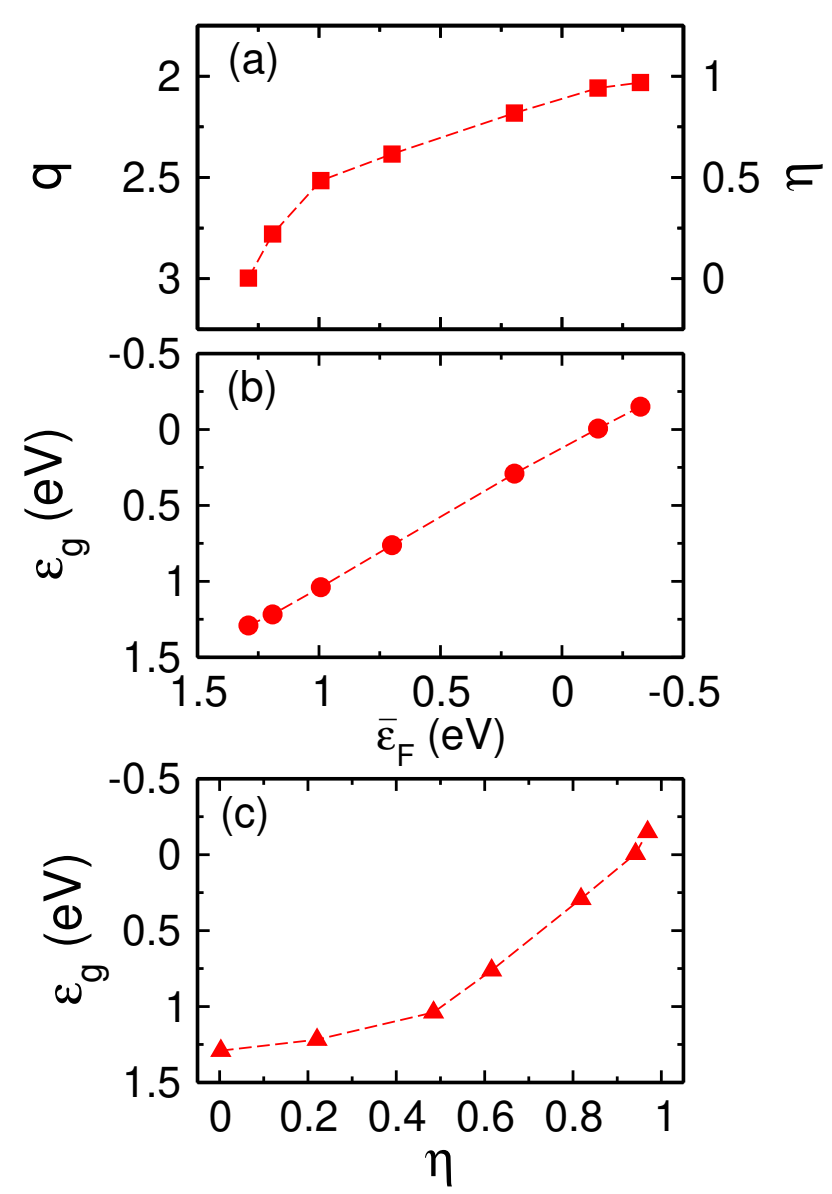

Fig. 2 Constant Fermi-level molecular dynamics of the aqueous $\mathrm{Fe}^{+2} / \mathrm{Fe}^{+3}$ redox couple: (a) evolution of the electronic charge (left axis) and of the occupation number (right axis) as a function of the preset Fermi energy $\bar{\epsilon}_{\mathrm{F}}$; (b) evolution of the single-particle energy level $\epsilon_{g}$ as a function of $\bar{\epsilon}_{\mathrm{F}}$; (c) $\epsilon_{g}$ as a function of occupation number $\eta$. The energies are referred to the SHE.

within at most $0.02 \mathrm{eV}$. However, the charge dynamics are slower and require longer equilibration times (at most $\sim 6 \mathrm{ps}$ ). The charge fluctuations depend directly on the choice of the fictitious mass parameter $M_{e}$ and the fictitious electronic temperature $T_{e}$.

Statistical averages are taken over the equilibrated parts of the trajectories and correspond to periods of at least $10 \mathrm{ps}$. Figure 2a shows the charge transition as a function of the preset value of the Fermi energy $\bar{\epsilon}_{\mathrm{F}}$. Through the appearance of fractional charges, the redox reaction is driven towards the formation of $\mathrm{Fe}^{+2}$. We remark that the charge of the system is fully determined by the occupation $\eta$ of the single-particle energy level in the gap, which is the only state undergoing 
an occupation change in our simulation. During this evolution, the single-particle energy level $\epsilon_{g}$ practically corresponds to $\bar{\epsilon}_{\mathrm{F}}$ (cf. Figure $2 \mathrm{~b}$ ), the small differences resulting from the width of the employed Gaussian smearing. From Figures 2a and $2 \mathrm{~b}$, it is possible to extract the dependence of $\epsilon_{g}$ on $\eta$ (Figure 2c).

The commonly used method to compute redox levels is thermodynamic integration. Within this technique the redox level is given by:

$$
\mu\left(q / q^{\prime}\right)=\frac{\Delta A}{q^{\prime}-q}+\frac{E_{\mathrm{corr}}^{q}-E_{\mathrm{corr}}^{q^{\prime}}}{q^{\prime}-q}-\epsilon_{\mathrm{v}},
$$

where $\epsilon_{\mathrm{v}}$ is the valence band maximum of the bulk system, $E_{\mathrm{corr}}^{q}$ an electrostatic finite-size correction term, and $\Delta A$ the thermodynamic integral given by:

$$
\Delta A=\int_{0}^{1}\langle\Delta E\rangle_{\eta} d \eta
$$

where $\eta$ is the Kirkwood coupling parameter and $\langle\Delta E\rangle_{\eta}$ is the total energy difference between charge states $q$ and $q^{\prime}$ averaged over configurations achieved at fixed $\eta$.

We here resort to Janak's theorem [30], according to which the derivative of the DFT energy functional $E[N]$ with respect to the occupation $f$ of the highest occupied Kohn-Sham (KS) state $g$ gives the KS eigenvalue $\epsilon_{g}$ corresponding to that state:

$$
\frac{\partial E[N]}{\partial f}=\epsilon_{g}
$$

In its integral form, Janak's theorem gives the change in energy of the system upon addition of one electron at fixed geometry,

$$
E^{N+1}-E^{N}=\int_{0}^{1} \epsilon_{g}(f) d f .
$$

The constant-Fermi-level technique consists in driving the electronic charge from state $q$ to state $q^{\prime}$ through the realization of several intermediate systems at fixed Fermi level. Although it is the Fermi level that is controlled during the simulations, the parameters of the simulation can be set to allow only for minimal fluctuations of the electronic charge of the system. Then, taking the electronic charge of the system as Kirkwood coupling parameter allows one to draw a clear similarity with the TI method. We assume that during the reaction a single state $g$ is involved and that its occupation goes from 0 to 1 . The electronic charge then corresponds to the occupation $\eta$ of state $g$. In this description, the TI method gives the redox level through the calculation of the thermodynamic integral $\Delta A$, as given in eq 5 . Using Janak's theorem in eq 7, the thermodynamic integral can be expressed as:

$$
\Delta A=\int_{0}^{1}\langle\Delta E\rangle_{\eta} d \eta=\int_{0}^{1}\left\langle\int_{0}^{1} \epsilon_{g}(f) d f\right\rangle_{\eta} d \eta=\int_{0}^{1} \int_{0}^{1} \epsilon(f, \eta) d f d \eta
$$

where we have used the following definition: 


$$
\epsilon(f, \eta)=\left\langle\epsilon_{g}(f)\right\rangle_{\eta}
$$

In the latter expression, the average is taken over structures corresponding to a state $g$ with occupation $\eta$, while $f$ indicates the occupation of $g$ at fixed geometry.

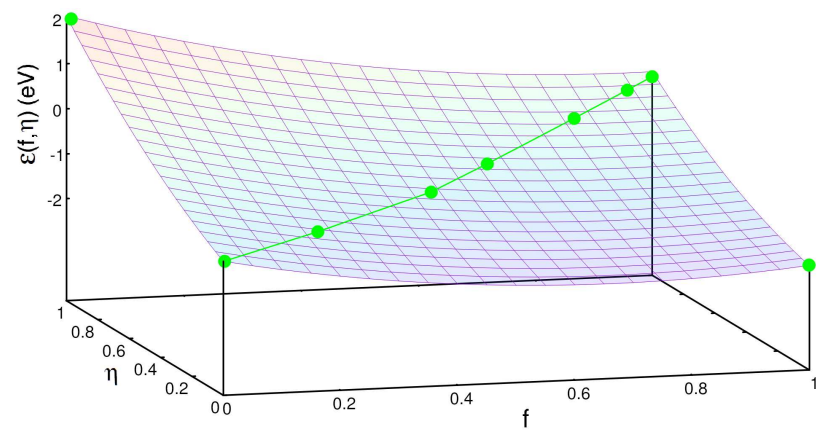

Fig. 3 Schematic representation of the function $\epsilon(f, \eta)$ in the twodimensional $(f, \eta)$ space. For capturing the quadratic dependence of $\epsilon(f, \eta)$, the dots shown in green are sampled in the present work.

As seen above, $\epsilon(f, \eta)$ is not necessarily a linear function. A more accurate approximation then consists in assuming a quadratic functional form in both $\eta$ and $f$ :

$$
\epsilon(f, \eta)=\epsilon_{\mathrm{S}}+\alpha\left(\eta-\frac{1}{2}\right)+\beta\left(f-\frac{1}{2}\right)+\alpha^{\prime}\left(\eta-\frac{1}{2}\right)^{2}+\beta^{\prime}\left(f-\frac{1}{2}\right)^{2}+\gamma\left(\eta-\frac{1}{2}\right)\left(f-\frac{1}{2}\right),
$$

where $\epsilon_{\mathrm{S}}, \alpha, \beta, \alpha^{\prime}, \beta^{\prime}$ and $\gamma$ are constants. Substituting eq 10 into eq 8 and carrying out the integrals leads to the following expression for the thermodynamic integral:

$$
\Delta A=\int_{0}^{1} \int_{0}^{1} \epsilon(f, \eta) d f d \eta=\epsilon_{\mathrm{S}}+\frac{\alpha^{\prime}+\beta^{\prime}}{12} .
$$

To determine $\epsilon_{\mathrm{S}}, \alpha^{\prime}$ and $\beta^{\prime}$, we propose to calculate $\epsilon(f, \eta)$ in the following cases. First, it is natural to determine $\epsilon(f, \eta)$ for $f=\eta$ as this follows directly from the constant Fermi level simulations:

$$
\begin{aligned}
\epsilon(\eta, \eta) & =\epsilon_{\mathrm{S}}+\underbrace{(\alpha+\beta)}_{A}\left(\eta-\frac{1}{2}\right)+\underbrace{\left(\alpha^{\prime}+\beta^{\prime}+\gamma\right)}_{B}\left(\eta-\frac{1}{2}\right)^{2} \\
& =\epsilon_{\mathrm{S}}+A\left(\eta-\frac{1}{2}\right)+B\left(\eta-\frac{1}{2}\right)^{2},
\end{aligned}
$$

where the parameters $\epsilon_{\mathrm{S}}, A$, and $B$ are easily obtained from a quadratic fit of $\epsilon(\eta, \eta)$. However, this fit is not sufficient to determine $\Delta A$ in eq 11 . Therefore, we consider two additional points in the twodimensional $(f, \eta)$ space: 


$$
\begin{aligned}
& \epsilon(0,1)=\epsilon_{\mathrm{S}}-\frac{1}{2}(\alpha-\beta)+\frac{1}{4}\left(\alpha^{\prime}+\beta^{\prime}-\gamma\right), \\
& \epsilon(1,0)=\epsilon_{\mathrm{S}}+\frac{1}{2}(\alpha-\beta)+\frac{1}{4}\left(\alpha^{\prime}+\beta^{\prime}-\gamma\right) .
\end{aligned}
$$

Combining eqs 12,13 , and 14 with eq 11 , we can express the thermodynamical integral as:

$$
\Delta A=\int_{0}^{1} \int_{0}^{1} \epsilon(f, \eta) d f d \eta=\frac{5}{6} \epsilon_{\mathrm{S}}+\frac{B}{24}+\frac{\epsilon(0,1)+\epsilon(1,0)}{12} .
$$

If $\epsilon(f, \eta)$ is given by a quadratic function, the present expression for the thermodynamic integral leads to the exact value of the redox level defined in eq 4. Figure 3 schematically illustrates the function $\epsilon(f, \eta)$ in the two dimensional $(f, \eta)$ space and the points sampled in the present scheme.

We remark that the presently adopted second-order form (eq 10) goes beyond Marcus' linear regime [31, 32]. Indeed, on the basis of Janak's theorem, the integration of $\epsilon(f, \eta)$ over $f$ produces the vertical energy gaps $\langle\Delta E\rangle_{\eta}$ required in the TI method (eq 5):

$$
\langle\Delta E\rangle_{\eta}=\int_{0}^{1} \Delta \epsilon(f, \eta) d f=\epsilon_{\mathrm{S}}+\alpha\left(\eta-\frac{1}{2}\right)+\alpha^{\prime}\left(\eta-\frac{1}{2}\right)^{2}+\frac{\beta^{\prime}}{12},
$$

which by construction accounts for one higher order more than Marcus' linear regime. However, we note that having recourse to a quadratic form for $\epsilon(f, \eta)$ is not inherent to the constant Fermi level approach. Indeed, when strong nonlinearities occur, higher order forms should be fitted to the data. Consequently, this entails a more detailed sampling of the function $\epsilon(f, \eta)$, in analogy to the more detailed sampling required for the vertical energy gaps in the TI method.

This procedure is applied to the determination of the redox potentials of aqueous ions. We carry out a detailed comparison between the redox potentials as obtained through the TI method and as extracted from the constant Fermi level simulations for three redox pairs: $\mathrm{Fe}^{3+} / \mathrm{Fe}^{2+}, \mathrm{O}_{2} \mathrm{H}^{-} / \mathrm{O}_{2} \mathrm{H}^{\bullet}$, and $\mathrm{MnO}_{4}^{2-} / \mathrm{MnO}_{4}^{-}$.

The simulation details described for the $\mathrm{Fe}^{3+} / \mathrm{Fe}^{2+}$ were also adopted for the other redox pairs. However, to account for the larger solute sizes, we used 31 and 28 water molecules in the simulation cell for $\mathrm{O}_{2} \mathrm{H}^{-} / \mathrm{O}_{2} \mathrm{H}^{\bullet}$ and $\mathrm{MnO}_{4}^{2-} / \mathrm{MnO}_{4}^{-}$, respectively. For each redox pair, we performed constant Fermi-level simulations at fixed $\bar{\epsilon}_{\mathrm{F}}$ corresponding to various values $\eta$ of occupation of the state $g$. These simulations could be used directly for determining the redox potentials through the TI method. For the configurations obtained during the molecular dynamics at a given $\eta$, we calculated the average total energy difference $\langle\Delta E\rangle_{\eta}$ between occupations $f=1$ and $f=0$. Integration of $\langle\Delta E\rangle_{\eta}$ yielded the thermodynamic integral $\Delta A$, from which the redox potential was derived through eq 4 . This result is taken as reference value in the following. The obtained values are listed in Table 1.

Following the scheme outlined previously, we also determined the redox potentials via the averaged Kohn-Sham eigenvalues $\epsilon(f, \eta)$ of state $g$. The constant Fermi-level simulation provided us directly with $\epsilon(\eta, \eta)$. Within the quadratic approximation, we 


\begin{tabular}{crrr} 
System & CFL & \multicolumn{1}{c}{ TI } & Expt. \\
\hline $\mathrm{O}_{2} \mathrm{H}^{\bullet} / \mathrm{O}_{2} \mathrm{H}^{-}$ & 0.47 & 0.42 & 0.75 \\
$\mathrm{Fe}^{+2} / \mathrm{Fe}^{+3}$ & 0.85 & 0.77 & 0.77 \\
$\mathrm{MnO}_{4}^{-} / \mathrm{MnO}_{4}^{-2}$ & -0.31 & -0.28 & 0.56
\end{tabular}

Table 1 Redox potentials vs. SHE (in eV) as obtained through constant Fermi level (CFL) simulations in the quadratic approximation, compared to those achieved with the thermodynamic integration (TI) method. Experimental values are shown for comparison.

additionally calculated $\epsilon(0,1)$ and $\epsilon(1,0)$. and yielded through eq 15 an improved description of the thermodynamic integrals. The corresponding redox potentials are given in Table 1. Comparison with the reference TI values shows agreement within $0.08 \mathrm{eV}$, validating the proposed scheme for the calculation of redox potentials.

All redox couples give redox levels falling inside the reduced band gap achieved in the present DFT calculations. The calculated redox potentials can thus be compared with their experimental counterparts at $300 \mathrm{~K}$ (Table 1). Considering the reference results obtained with the TI method, we found an agreement with experiment within $0.3 \mathrm{eV}$ for $\mathrm{O}_{2} \mathrm{H}^{\bullet} / \mathrm{O}_{2} \mathrm{H}^{-}$and $\mathrm{Fe}^{+2} / \mathrm{Fe}^{+3}$ and a larger deviation of 0.84 $\mathrm{eV}$ for $\mathrm{MnO}_{4}^{-} / \mathrm{MnO}_{4}^{-2}$. This level of agreement can be considered typical for DFT schemes at the present level of theory, as errors up to $0.9 \mathrm{eV}$ have been found in redox potentials calculated with GGA functionals in previous studies [33, 34]. In particular, the large deviation observed for the permanganate redox potential could in part also arise from the choice of the volume that we allocated to the $\mathrm{MnO}_{4}^{-}$ion, in the absence of any estimate of its partial molar volume.

\section{CFL-MD at metal-water interface: The case of the Volmer reaction}

In this section, we apply the constant Fermi energy molecular dynamic to the metalwater interface. In particular we focus on the reaction mechanism of the Volmer reaction. Our interface model consists of a $3 \times 4 \mathrm{Pt}$ slab of 3 layers, 31 water molecules, and one hydronium ion within a supercell of $9.79 \times 8.48 \times 19.50 \AA^{3}$. This size was chosen to ensure a water density of $1 \mathrm{~g} / \mathrm{cm}^{3}$ and a bulk-like $g_{\mathrm{OO}}(r)$ partial correlation function. The adopted computation setup is similar to that used for the study of aqueous ions and is detailed in ref [35].

We applied constant Fermi level MD to the Pt(111)/water interface by setting the Fermi level at $U=-0.88 \mathrm{eV}$ and $U=-0.92 \mathrm{eV}$ within the band gap of liquid water. Figure 4 represents the time evolution of the total electronic charge $Q_{\text {tot }}$ of the system. We remark that $Q_{\text {tot }}$ exhibits a jump at 6.2 and 4.3 ps for $U=-0.88$ and $-0.92 \mathrm{eV}$, respectively. This change in the total electronic charge corresponds to the adsorption of the $\mathrm{H}_{\mathrm{aq}}^{+}$ion at the $\mathrm{Pt}$ surface and signals electron transfer from the electron reservoir to the electrode according to the Volmer reaction: $\mathrm{H}_{\mathrm{aq}}^{+}+\mathrm{e}^{-} \rightarrow \mathrm{H}_{\mathrm{ad}}$. 


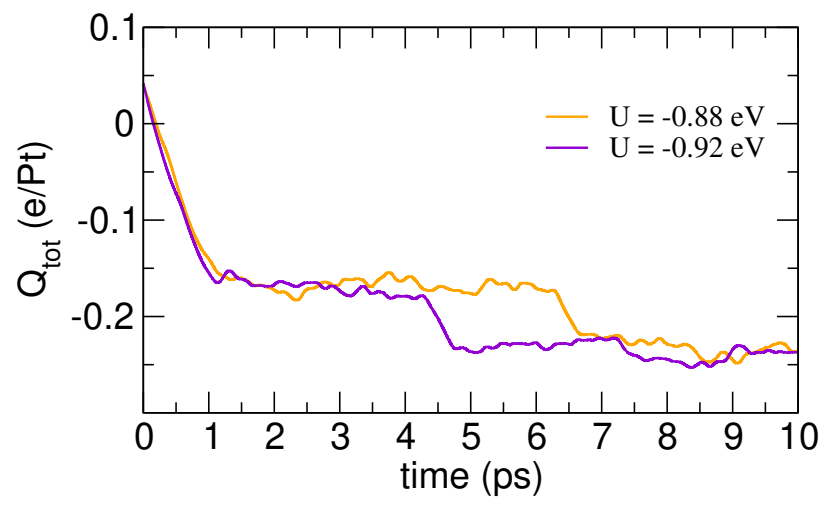

Fig. 4 Time evolution of the total electronic charge $Q_{\text {tot }}$ in constant Fermi-level molecular dynamics of the $\operatorname{Pt}(111) /$ water interface at an electrode potential of $U=-0.88 \mathrm{eV}$ and $U=-0.92$. $Q_{\text {tot }}$ is defined as the total electronic charge and is here given per number of Pt surface atoms.

This reaction is expected to occur stochastically for electrode potentials lower than the SHE level provided that the simulation time is long enough.
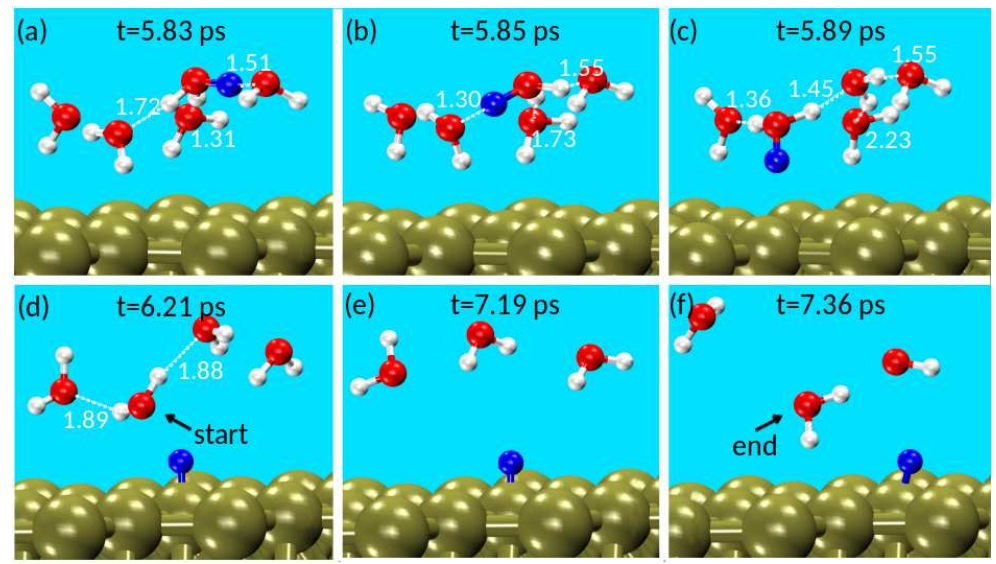

Fig. 5 (a-d) Representative snapshots illustrating the atomistic mechanism of the Volmer reaction, in which the Eigen complex $\mathrm{H}_{9} \mathrm{O}_{4}^{+}$transforms into $\mathrm{a}_{7} \mathrm{O}_{3}^{+}$complex before releasing the $\mathrm{H}$ atom to the Pt surface at $U=-0.88 \mathrm{eV}$. (e-f) Snapshots illustrating the reorientation of the water molecule after $\mathrm{H}$ adsorption. The distances between $\mathrm{H}$ and $\mathrm{O}$ atoms (dashed white line) are given in $\AA$.

By inspecting the atomistic structure we find in our simulations that the hydronium diffusion in bulk water is mediated by an Eigen complex $\mathrm{H}_{9} \mathrm{O}_{4}^{+}$. Figure 5 shows the reaction mechanism of the Volmer reaction, as obtained for $U=-0.88 \mathrm{eV}$. This reaction occurs in three steps. First, the $\mathrm{H}_{9} \mathrm{O}_{4}^{+}$complex diffuses into the vicinity of the Pt interface [Fig. 5(a-b)]. Second, the proton is transferred to a water molecule 


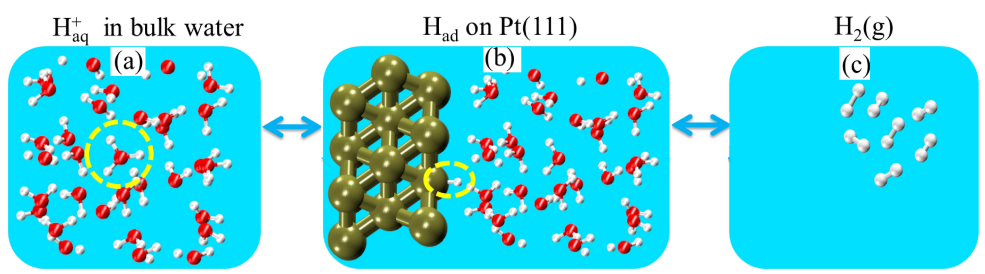

Fig. 6 Schematic representation of the three steps of the standard hydrogen reaction considered in our work.

in a $\mathrm{H}_{\text {down }}$ configuration and the Eigen complex transforms to a $\mathrm{H}_{7} \mathrm{O}_{3}^{+}$complex [Fig. 5(c)]. Third, the proton is adsorbed at the metal surface [Fig. 5(e)] leaving the water molecule in a $\mathrm{O}_{\text {down }}$ configuration, which is energetically unfavorable. This leads to the reorientation of this water molecule resulting in a $\mathrm{H}_{\text {down }}$ configuration within a time span of $\sim 1.15 \mathrm{ps}$. We note that the same reaction mechanism occurs at $U=-0.92 \mathrm{eV}$. Otani et al. also investigated the Volmer reaction varying the electrode potential though the introduction of excess charge [13]. In their work, the hydronium ion was found to diffuse through a $\mathrm{H}_{5} \mathrm{O}_{2}^{+}$complex. The involvement of a larger complex in our simulation might be due to the account of the van der Waals interactions, which strongly impact the hydrogen network. Nevertheless, the reported reorientation mechanism and its duration of $\sim 0.8 \mathrm{ps}$ are confirmed by our findings [13].

\section{Referencing the bias potential to the SHE}

One of the most difficult tasks in modelling electrified metal/water interface is to properly reference the electrode potential $U$ to the SHE. We here define the electrode potential $\tilde{U}$ on a non-inverted scale and referenced to the valence band maximum of liquid water $\left(\epsilon_{\mathrm{vbm}}\right)$ according to: $\tilde{U}=-U+\mu_{\mathrm{SHE}}-\epsilon_{\mathrm{vbm}}$.

We define the SHE as a sequence of two reactions (see fig. 6 in which the intermediate state corresponds to a neutral hydrogen atom $\mathrm{H}_{\mathrm{ad}}$ adsorbed at the $\mathrm{Pt}$ surface:

$$
\begin{gathered}
\mathrm{H}_{\mathrm{aq}}^{+}+\mathrm{e}^{-} \rightarrow \mathrm{H}_{\mathrm{ad}}, \\
\mathrm{H}_{\mathrm{ad}} \rightarrow \frac{1}{2} \mathrm{H}_{2}(\mathrm{~g})+\mathrm{Pt} .
\end{gathered}
$$

We obtain the Gibbs free energy difference $\Delta G$ of the full reaction as the sum of $\Delta G_{1}$ and $\Delta G_{2}$, which correspond to reactions (17) and (18), respectively.

To express $\Delta G_{1}$, we use the grand canonical formulation for describing solutes and defects $[36,28]$ :

$$
\Delta G_{1}=G\left(\mathrm{H}_{\mathrm{ad}}\right)-G\left(\mathrm{H}_{\mathrm{aq}}^{+}\right)-\left(\epsilon_{\mathrm{vbm}}+\mu_{\mathrm{e}}\right)
$$



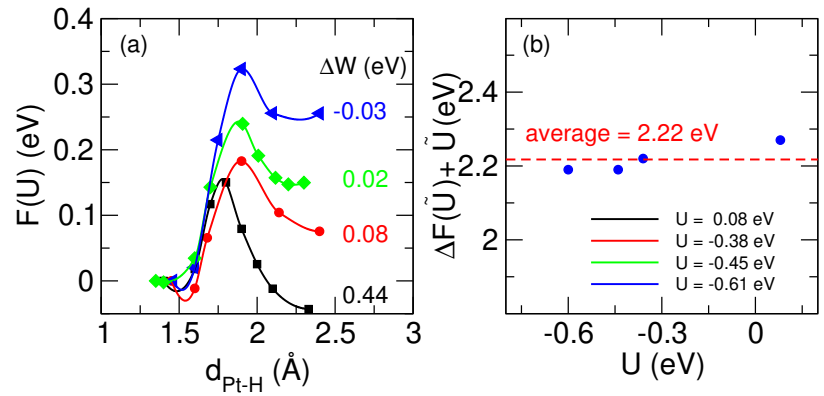

Fig. 7 (a) Free energy profiles and (b) $\Delta F(\tilde{U})+\tilde{U}$ as obtained through Blue Moon simulations for various electrode potentials expressed in terms of $U$. In (a), we also give the potential difference $\Delta W$ between the contact layer and the bulk-like region for each simulation.

where $\mu_{\mathrm{e}}$ is the electron chemical potential. In our work, the difference $G\left(\mathrm{H}_{\mathrm{ad}}\right)-$ $G\left(\mathrm{H}_{\mathrm{aq}}^{+}\right)$is evaluated within the constant Fermi level method, in which the energy is governed by the grand canonical potential $\Omega_{\tilde{U}}=E^{\mathrm{tot}}\left(r_{i}, N_{\mathrm{e}}\right)-N_{\mathrm{e}}\left(\epsilon_{\mathrm{vbm}}+\tilde{U}\right)$, where $E^{\text {tot }}$ is the DFT total energy and $N_{\mathrm{e}}$ the number of electrons in the system. Here, the Gibbs free energies actually correspond to Helmholtz free energies, as volume variations are small and can be neglected [28]. We use the notation $F(\tilde{U})$ to indicate the free energy associated to the potential $\Omega_{\tilde{U}}$. Thus, we write:

$$
\begin{aligned}
\Delta G_{1} & =F_{\tilde{U}}\left(\mathrm{H}_{\mathrm{ad}}\right)-F_{\tilde{U}}\left(\mathrm{H}_{\mathrm{aq}}^{+}\right)+\tilde{U}+\Delta_{\mathrm{zp}}^{\mathrm{H}_{\mathrm{ad}}}-\Delta_{\mathrm{zp}}^{\mathrm{H}_{\mathrm{aq}}^{+}}-\mu_{\mathrm{e}} \\
& =\Delta F(\tilde{U})+\tilde{U}+\Delta_{\mathrm{zp}}^{\mathrm{H}_{\mathrm{ad}}}-\Delta_{\mathrm{zp}}^{\mathrm{H}_{\mathrm{aq}}^{+}}-\mu_{\mathrm{e}},
\end{aligned}
$$

where we have isolated the zero point energies of $\mathrm{H}_{\mathrm{ad}}$ and $\mathrm{H}_{\mathrm{aq}}^{+}$, i.e. $\Delta_{\mathrm{zp}}^{\mathrm{H}_{\mathrm{ad}}}$ and $\Delta_{\mathrm{zp}}^{\mathrm{H}_{\mathrm{aq}}^{+}}$, as they are not explicitly accounted for in our DFT framework. $\Delta_{\mathrm{zp}}^{\mathrm{H}_{\mathrm{aq}}^{+}}$amounts to $0.36 \mathrm{eV}$ [28], while $\Delta_{\mathrm{zp}}^{\mathrm{H}}$ does not need to be evaluated as it drops in our final expressions for SHE. In Eq. (20), we introduced the free energy difference $\Delta F(\tilde{U})=F\left(\mathrm{H}_{\mathrm{ad}}\right)-F\left(\mathrm{H}_{\mathrm{aq}}^{+}\right)$ as described in our constant Fermi energy method. We determine $\Delta F(\tilde{U})$ through the Blue Moon scheme following recent literature [37, 38], in which redox reaction mechanisms at constant bias potential were studied. We use constrained molecular dynamics, in which the distance $z$ between the proton and the Pt surface is taken as reaction coordinate $[39,40]$ :

$$
\Delta F(\tilde{U})=\int\left\langle f_{\mathrm{c}}(z)\right\rangle d z
$$

where $f_{\mathrm{c}}(z)$ is the constraint force computed within the constant Fermi energy scheme. Hence, the number of electrons in the system $N_{e}$ depends on the spatial coordinate $z$. 
The reaction coordinate in Eq. (21) goes from the position of the adsorbed $\mathrm{H}_{\mathrm{ad}}$ to the bulk-like water region. In practice, we perform the Blue Moon simulation until the hydrogen atom reaches the first contact layer, where it is found in a singly positive charge state, and the remaining part of the integral is evaluated by considering the potential difference $\Delta W$ between the contact layer and the bulk-like water region. The latter is easily determined through the variation of $\mathrm{O}_{2 s}$ levels and is given in Fig. 7(a). In Fig. 7(a), we show the obtained free energy profiles for four different values of $\tilde{U}$, equivalently expressed in terms of $U$ in the figure. $\Delta F(\tilde{U})+\tilde{U}$ should not depend on the value of $\tilde{U}$, and this is confirmed by the results of our simulations in Fig. 7(b), where its average value is shown to differ by less than $0.05 \mathrm{eV}$ from the individual simulations. This result corroborates our scheme and supports its consistency.

For the second reaction (18), the free energy difference is given by:

$$
\Delta G_{2}=G\left[\frac{1}{2} \mathrm{H}_{2}(\mathrm{~g})\right]+G(\mathrm{Pt})-G\left(\mathrm{H}_{\mathrm{ad}}\right)=\mu_{\mathrm{H}}+G(\mathrm{Pt})-G\left(\mathrm{H}_{\mathrm{ad}}\right),
$$

where $\mu_{\mathrm{H}}$ is the hydrogen chemical potential set to the free energy of gaseous $\mathrm{H}_{2}$ in standard conditions and is evaluated as described in Ref. [28]. In our work, we express $\Delta G_{2}$ as:

$$
\Delta G_{2}=\mu_{\mathrm{H}}+\Delta A_{\mathrm{TI}}-\Delta_{\mathrm{zp}}^{\mathrm{H}_{\mathrm{ad}}}
$$

where $\Delta A_{\mathrm{TI}}$ corresponds to the free energy cost of removing the $\mathrm{H}_{\mathrm{ad}}$ from the $\mathrm{Pt}$ surface and placing it in the vacuum without considering the zero point energy of $\mathrm{H}_{\mathrm{ad}}$, which is expressed separately. We remark that, $\Delta G_{2}$ does not depend on the electrode potential because there is no charge transfer involved in the reaction.

We evaluate $\Delta A_{\mathrm{TI}}$ through the thermodynamic integration method, in which the Kirkwood parameter $\eta$ evolves from $\eta=0$ in the initial state with $\mathrm{H}_{\mathrm{ad}}$ on the Pt surface to $\eta=1$ in the final state in which the hydrogen atom has been removed. At $\eta=0$, the vertical energy difference is obtained by removing the $\mathrm{H}$ atom from a MD run, in which $\mathrm{H}_{\mathrm{ad}}$ is attached to the Pt surface in aqueous environment. At $\eta=1$, the $\mathrm{H}$ atom is vertically inserted in configurations from a MD of the pristine Pt/water interface and allowed to relax while keeping all other atoms at fixed positions. We use two points at $\eta=0$ and $\eta=1$ to compute $\Delta A_{\mathrm{TI}}+\mu_{\mathrm{H}}$. We find that the vertical energy differences do not differ by more than $0.1 \mathrm{eV}$ in the two cases, indicating that more intermediate values of $\eta$ are not necessary for an accurate result. This behaviour should be ascribed to the fact that the reaction only involves neutral species and that the $\mathrm{H}$ occupies a position within the empty layer that separates the Pt surface from the water molecules. The integration of $\Delta A_{\mathrm{TI}}+\mu_{\mathrm{H}}$ over $\eta$ yields $0.21 \mathrm{eV}$.

The redox level $\mu_{\mathrm{e}}$ for which $\Delta G=\Delta G_{1}+\Delta G_{2}=0$ can be isolated and gives:

$$
\mu_{\mathrm{e}}=\Delta F(\tilde{U})+\tilde{U}+\Delta A_{\mathrm{TI}}+\mu_{\mathrm{H}}-\Delta_{\mathrm{zp}}^{\mathrm{H}_{\mathrm{aq}}^{+}} .
$$

We remark that this redox level has been calculated for the neutral interface, which corresponds to the $\mathrm{pH}$ at the point of zero charge (pzc). To retrieve the SHE level, a $\mathrm{pH}=0$ should be used. This leads to the consideration of a Nernstian shift from the $\mathrm{pH}_{\mathrm{pzc}}$ to $\mathrm{pH}=0$ : 


$$
\mu_{\mathrm{e}}^{\mathrm{SHE}}=\mu_{\mathrm{e}}(\mathrm{pH}=0)=\mu_{\mathrm{e}}-(0.059 \mathrm{eV}) \cdot \mathrm{pH}_{\mathrm{pzc}},
$$

where $\mathrm{pH}_{\mathrm{pzc}}$ of the $\mathrm{Pt}(111) /$ water interface has experimentally been estimated at 3.4 [41, 42]. Combining Eqs. (24) and (25), we finally locate the SHE level at:

$$
\mu_{\mathrm{e}}^{\mathrm{SHE}}=\Delta F(\tilde{U})+\tilde{U}+\Delta A_{\mathrm{TI}}+\mu_{\mathrm{H}}-\Delta_{\mathrm{zp}}^{\mathrm{H}_{\mathrm{aq}}^{+}}-(0.059 \mathrm{eV}) \cdot \mathrm{pH}_{\mathrm{pzc}} .
$$

The SHE level calculated in this way falls at $1.87 \mathrm{eV}$ with respect to the valence band of liquid water, as determined with the rVV10 exchange correlation functional.

\section{Macroscopic properties at the metal-water interface}

We first focus on the electric field established at the Pt interface. We consider the metal/water interface model of size $L_{\mathrm{x}} \times L_{\mathrm{y}} \times L_{\mathrm{z}}$ shown in Figure 8 with the water layer centered at $z=0$. The boundary surfaces of the Pt electrode are at $z= \pm L_{\mathrm{w}} / 2$. In our simulation, we make use of a uniform neutralizing background charge $\left(\rho_{\mathrm{bg} 1}\right)$, as schematically illustrated in the top panel of Fig. 8. However, in the physical situation that we would like to model the compensating charge $\left(\rho_{\mathrm{bg} 2}\right)$ is found in the Helmholtz layer, as depicted in the bottom panel of Fig. 8. We account for these differences through classical electrostatics.

It should be realized that the uniform background entails effects on both the charge on the electrode and the potential difference $\Delta V$ between the electrode and bulk-like water. We first consider the effect on the charge. When the system is charged with a given nominal charge $Q_{1}$ on the electrode, a uniform background is established throughout the cell. Part of the added charge is directly compensated by the fraction of the uniform background charge that lies within the metal electrode. The actual charge $Q_{2}$ that contributes to the establishment of an electric field is therefore reduced, $Q_{2}=\eta Q_{1}$, where $\eta=L_{\mathrm{w}} / L_{\mathrm{z}}$ is the fractional volume occupied by the water component expressed in terms of the width of the water layer $L_{\mathrm{w}}$ and the cell size $L_{\mathrm{z}}$ in the $Z$ direction. In our DFT simulations $\eta=0.71$.

Next, to correct the difference in potential, we compare two classical models showing the same cells as in our DFT simulations. In the first one, the added charge is compensated by a uniform background charge $Q_{1}$, while the second one represents the physical model with charge $Q_{2}$ (Fig. 8). In the case of the a uniform background charge, we perform a simulation at a total net charge $Q_{1}$. An opposite charge is induced in the neutralizing background and is uniformly distributed over the cell leading to a charge density $\rho_{\mathrm{bg} 1}$ :

$$
\rho_{\mathrm{bg} 1}=\frac{-Q_{1}}{L_{x} L_{y} L_{z}} .
$$

The potential induced by this charge density is the solution of the Poisson equation: 


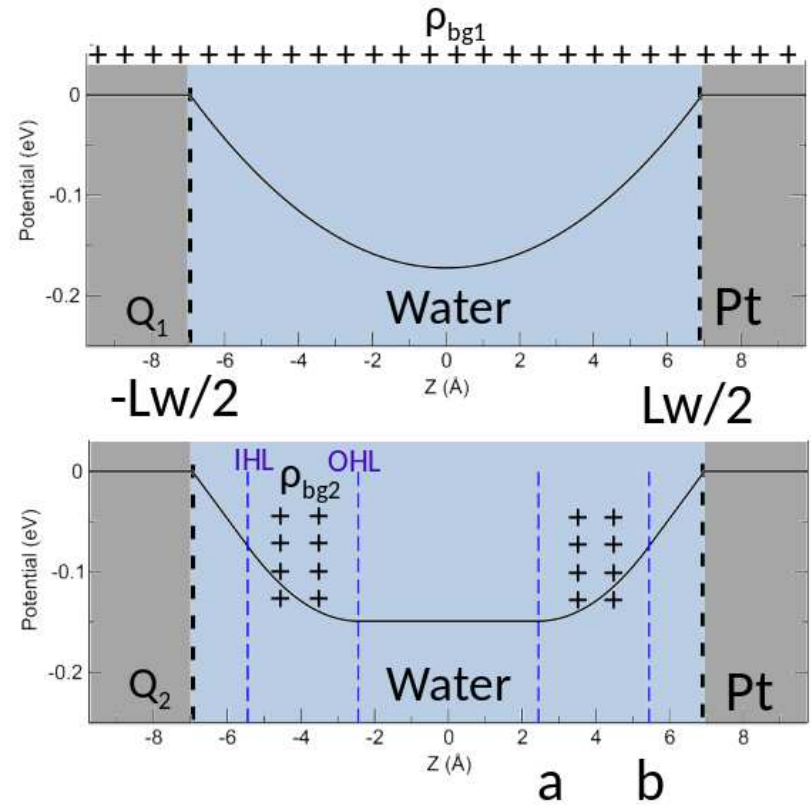

Fig. 8 Schematic representation of the metal/water interface in two different classical models. In the top panel, the extra charge $Q_{1}$ added on the electrode is compensated by a uniform background charge $\rho_{\text {bg } 1}$ like in DFT supercell simulations. In the bottom panel, the extra charge on the electrode $Q_{2}$ is compensated by a background charge $\rho_{\text {bg2 }}$ confined between the inner (IHL) and outer (OHL) Helmholtz layers. $Q_{2}$ corresponds to the noncompensated charge on the electrode when a charge $Q_{1}$ is added to the model with a uniform background. The calculated potential corresponds to the case in which $Q_{1}$ equals four electrons. The cells of the classical models correspond to that used in the DFT simulations.

$$
\nabla^{2} V=\frac{-4 \pi \rho_{\mathrm{bg} 1}}{\epsilon}
$$

Considering the boundary conditions $V\left(-L_{\mathrm{w}} / 2\right)=V\left(L_{\mathrm{w}} / 2\right)=0$, we find the following quadratic function for $V(z)$ :

$$
V(z)=\frac{-2 \pi \rho_{\mathrm{bg} 1} z^{2}}{\epsilon}+\frac{2 \pi \rho_{\mathrm{bg} 1}\left(L_{\mathrm{w}} / 2\right)^{2}}{\epsilon} .
$$

In the case of the double layer model, the corresponding situation is obtained by considering an additional charge $Q_{2}$, which leads to the following surface charge density at each Pt surface:

$$
\sigma_{2}=\frac{Q_{2}}{2 L_{x} L_{y}}
$$

and an opposite background charge confined in the double layer: 


$$
\rho_{\mathrm{bg} 2}=\frac{-Q_{2}}{2 L_{x} L_{y}(b-a)}
$$

Solving the Poisson equation in this system leads to the following potential:

$$
\begin{aligned}
& V(z)=\frac{-2 \pi \rho_{\mathrm{bg} 2}(a-b)^{2}}{\epsilon}+\frac{4 \pi \sigma_{2}\left(a-L_{\mathrm{w}} / 2\right)}{\epsilon} ; \text { for } z<a \\
& V(z)=\frac{-2 \pi \rho_{\mathrm{bg} 2}(z-b)^{2}}{\epsilon}+\frac{4 \pi \sigma_{2}\left(z-L_{\mathrm{w}} / 2\right)}{\epsilon} ; \text { for } a<z<b \\
& V(z)=\frac{4 \pi \sigma_{2}\left(z-L_{\mathrm{w}} / 2\right)}{\epsilon} ; \text { for } b<z<L_{\mathrm{w}} / 2
\end{aligned}
$$

We remark that the two models show very close electric fields in the region of the double layer, but the potentials bend to different values in the middle of the water region. Indeed, the model with a uniform background shows a parabolic behavior, whereas the physical one exhibits a flat potential after crossing the Helmholtz layer. In Fig. 8, these potentials have been calculated for a nominal charge $Q_{1}$ of four electrons. The screening of the water molecules is accounted for by a dielectric constant $\epsilon=62.4$, corresponding to the experimental value at $T=350 \mathrm{~K}$ (Ref. [43]). In these conditions, the model with the uniform background charge yields $\Delta V_{1}=0.17 \mathrm{eV}$, to be compared with $\Delta V_{2}=0.15 \mathrm{eV}$ in the physical model. The difference between these values should be used to correct the DFT potential.

For the particular simulation cell considered in our work, the numerical value of this difference is negligible. Hence, in our simulation, apart from the nominal correction of the extra charge on the electrode, the background charge does not produce any sizeable effect on the alignment. This implies that the uniform background effectively accounts for the counterions in an averaged way. However, we note that in general such corrections depend on the width of the water layer in the simulation cell.

We now focus on two electrochemical properties of the $\operatorname{Pt}(111) /$ water interface system, the double layer capacitance and the potential of zero charge. We applied constant Fermi level MD to the Pt(111)/water interface by setting the Fermi level at various positions within the band gap of liquid water. Within the alignment scheme detailed above, our applied potential covers a range from -0.92 to $0.44 \mathrm{eV}$ versus the SHE. We define the excess charge at Pt surface $\mathrm{Q}_{\mathrm{dl}}$ as $Q_{\mathrm{dl}}=\frac{1}{2}\left(\eta Q_{\mathrm{tot}}-1\right)$, where $\eta$ is the fractional volume corresponding to water in the supercell and accounts for the passivation of the background charge in the metal slab. In this definition, it is assumed that the aqueous hydronium ion $\mathrm{H}_{\mathrm{aq}}^{+}$always carries a positive charge and that the electronic charge is shared between the two Pt interfaces in the cell.

Figure 9 presents the evolution of the double layer charge as a function of the electrode potential. The pzc corresponds to the Fermi level of the neutral system with respect to the SHE level and is found at $0.22 \mathrm{eV}$ in our simulation, in agreement with reported experimental values (0.28-0.37 eV vs. SHE [42, 44, 41]). Another accessible macroscopic electrochemical quantity is the double layer capacitance $C_{\mathrm{dl}}=d Q_{\mathrm{dl}} / d U$, and is compared to respective measured data in Fig. 9. The dependence of the calculated capacitance on $U$ reproduces well the shape of the 


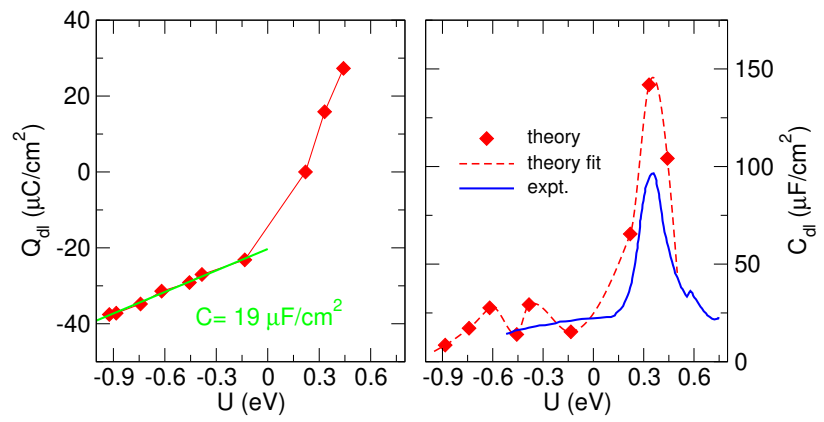

Fig. 9 (left panel) Double layer charge $Q_{\mathrm{dl}}$ as a function of the applied electrode potential $U$. The red line represents a linear fit, from which the double layer capacitance $C_{\mathrm{dl}}$ is derived. (right panel) Double layer capacitance (blue dots) as a function of the applied electrode potential $U$. The simulation data are fitted through a polynomial function (blue dashed line) and are compared to experimental results (green line) from Ref. [44]. The energies are referred to the SHE.

experimental data, and yields $C_{\mathrm{dl}} \simeq 19 \mu \mathrm{Fcm}^{-2}$, in excellent agreement with the commonly accepted experimental value of $\sim 20 \mu \mathrm{Fcm}^{-2}$ (Refs. [45, 46] and [44]) We remark that the main peak in the $C_{\mathrm{dl}}$ at $U \simeq 0.33 \mathrm{eV}$ is associated with a rapid change in the double layer capacitance, which in turn is linked to the atomic-scale structural reorganization of the electrical double layer, as will be discussed in the analysis below. These results demonstrate the predictive power of our modelling scheme in accessing macroscopic electrochemical properties.

\section{Atomic-scale processes at the metal-water interface}

The atomic-scale structure of the electrical double layer is an important aspect in defining the metal/water interface and the electrochemical activity of the electrode [47]. Several experimental studies investigated the structure at interfacial water systems, either in neutral conditions or under bias potential. Water molecules have been observed to undergo reorientation depending on the applied potential in a variety of systems, including $\operatorname{Pt}(111)$ [48, 49], polycrystalline Pt [50], $\mathrm{Au}(111)$ [51], air/water and lipid/water charged interfaces. In particular, at metal surfaces, the interfacial water molecules are strongly hydrogen bonded with the $\mathrm{O}$ atoms pointing toward the metal surface for potentials above the pzc. At variance, for potentials below the pzc, the water molecules are weakly bonded and point their $\mathrm{H}$ atoms downwards. In this work, we take advantage of our theoretical scheme to study the structural reorganization of the water double layer at the $\mathrm{Pt}(111) / \mathrm{H}_{2} \mathrm{O}$ interface as a function of the bias electrode potential referenced to the SHE.

Figure 10 shows the distribution of $\mathrm{O}$ and $\mathrm{H}$ atoms, and the water dipole orientation as a function of a coordinate along the surface normal direction as the electrode potential $U$ is varied. For electrode potentials above the pzc $(U>0.22 \mathrm{eV})$, the $\mathrm{O}$ 


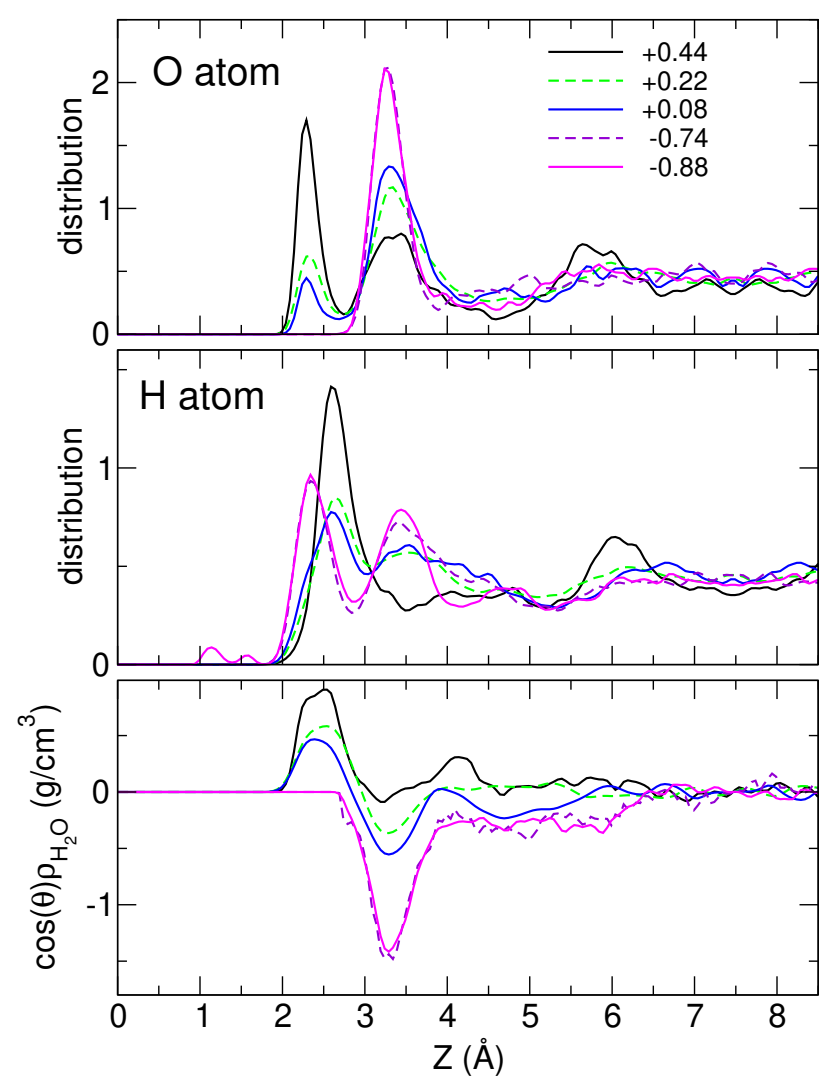

Fig. 10 Distribution of O (top panel) atoms, $\mathrm{H}$ (middle panel) atoms, and dipole orientation (bottom panel) as a function of the coordinate $Z$ oriented along the surface normal direction, at various electrode potentials (in $\mathrm{eV}$ ). The $Z$ coordinate is referenced with respect to the Pt surface. The distributions are obtained from an average over the two interfaces in our model and have been smoothed with a Gaussian function having a width of $0.05 \AA$.

distribution features a first sharp peak at $2.3 \AA$ and a second broad peak around $3.35 \AA$, while the $\mathrm{H}$ distribution exhibits a single principal peak centered at $2.6 \AA$. The orientation of the water molecules can be inferred from the dipole orientation, as presented in Figs. 10 and Fig. 11. The positive peak of the dipole distribution indicates that the water molecules in the first contact layer are in $\mathrm{O}_{\text {down }}$ configurations with the $\mathrm{H}$ atoms pointing towards the liquid. Specifically, we find two main dipole orientations at $55^{\circ}$ and $86^{\circ}$ for $U=0.44 \mathrm{eV}$ (see Fig. 11) The dipole orientation of the water molecules in the second layer shows a very broad peak around $90^{\circ}$ (see Fig. 11). As the potential decreases, the intensity of the first $\mathrm{O}$ peak diminishes, while that of the second peak increases (Fig. 10). This trend is accompanied by a reduction in the intensity of the first peak of the $\mathrm{H}$ distribution and the appearance of a second broad peak centered at $3.8 \AA$ (Fig. 10). Focusing on the dipole orientation, 
we infer that some of the water molecules in the first contact layer reorient from $\mathrm{O}_{\text {down }}$ to $\mathrm{H}_{\text {down }}$ configurations, as the electrode potential drops. At the pzc $(0.22 \mathrm{eV})$, we observe coexistence of the two configurations with a slight prevalence of the $\mathrm{H}_{\text {down }}$ configurations.
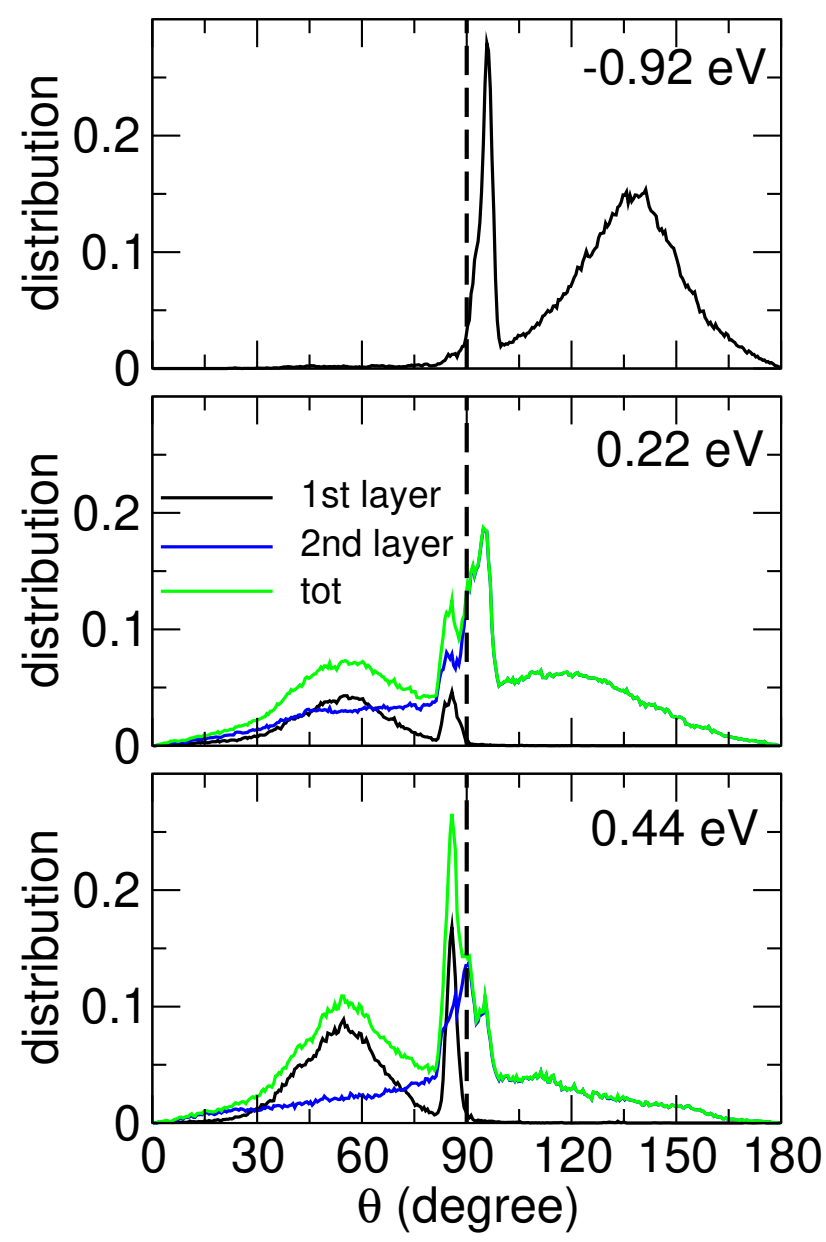

Fig. 11 The dipole angular distribution of water molecules belonging to the electrical double layer at electrode potentials $U=0.44 \mathrm{eV}$ (bottom panel), $0.22 \mathrm{eV}$ (middle panel), and $-0.92 \mathrm{eV}$ (top panel). The dashed black line corresponds to $\theta=90^{\circ}$.

Under negative bias, all the water molecules are found in $\mathrm{H}_{\text {down }}$ configurations leading to the disappearance of the first $\mathrm{O}$ peak in Fig. 10. In addition, the first peak in the $\mathrm{H}$ distribution shifts toward $2.35 \AA$ and a second $\mathrm{H}$ peak appears around $3.43 \AA$. The dipole orientation features a negative peak signifying that the water orientation is predominantly $\mathrm{H}_{\text {down }}$. A further broad negative region appearing under strong 
negative bias indicates that also the molecules in the second water layer $(4<Z<6 \AA)$ orient their $\mathrm{H}$ atoms toward the interface. Accordingly, two main dipole orientations are found at $96^{\circ}$ and $134^{\circ}$ at $U=-0.92 \mathrm{eV}$ (see Fig. Fig. 11). However, deep in the water layer $(Z>6.5 \AA)$ the dipole is found to be randomly oriented and the water structure can be taken as representative of the bulk phase. The atomistic description emerging from our work is fully consistent with the picture of water reorientation under bias potential inferred from experimental data [48, 51, 50, 49].

\section{Conclusion}

In this contribution we first reviewed the constant Fermi energy molecular dynamics. Then, we applied this technique to the determination of redox levels. We showed that the redox reaction can be driven through the control of the Fermi level without any prior knowledge of the reaction products. For aqua ions, we demonstrated molecular dynamics with stable evaluations of the Fermi level and of the electronic charge, without any divergence or instability. Through Janak's theorem, a relation with the redox potential could be established. Introducing a quadratic function to model the dependence of the single-particle energy level on occupation, we found agreement within less than $0.1 \mathrm{eV}$ with redox potentials calculated through the thermodynamic integration method.

Then we applied the constant Fermi-level molecular dynamics to study the $\mathrm{Pt}(111) /$ water interface under variable bias potential. First, we investigated the Volmer reaction mechanism involving the adsorption of a hydrogen atom at the Pt interface under negative bias. Second, we established an alignment scheme to determine the SHE reference level by considering the hydrogen adsorbed at the $\mathrm{Pt}$ surface as an intermediate step between the aqueous hydronium ion $\mathrm{H}_{\mathrm{aq}}^{+}$and the gas phase $\mathrm{H}_{2}(\mathrm{~g})$. Through the determination of the SHE level, our scheme allows one to reference the electrode potentials on an absolute scale. Third, we studied the double layer capacitance and the pzc, and found $\mu_{\mathrm{pzc}}=0.22 \mathrm{eV}$ and $C_{\mathrm{dl}} \simeq 19 \mu \mathrm{Fcm}^{-2}$, in very good agreement with experiment. Finally, we investigated the atomic-scale structural reorganization of the electrical double layer as a function of the bias potential. Our results reveal that at potentials above the pzc the water structure is dominated by $\mathrm{O}_{\text {down }}$ configurations, in which the dipole of the water molecules is on average pointing towards the bulk water layer. At the pzc, $\mathrm{H}_{\text {down }}$ configurations coexist with $\mathrm{O}_{\text {down }}$ structures, but the latter disappear completely at negative bias potential, for which all the water dipoles are fully oriented toward the Pt surface. At the lowest potential considered, the water molecules orient both their $\mathrm{H}$ atoms toward the electrode.

Overall, our modeling scheme gives simultaneously access to macroscopic and microscopic properties of aqueous species and metal/water interfaces. This technique will considerably contribute to advancing the predictive power of the DFT modelling of electrode/electrolyte interfaces under bias potential. 


\section{Acknowledgements}

This project has received funding from the European Union's Seventh Framework Programme for research, technological development and demonstration under grant agreement no. 291771 (call 2014). This work has been performed in the context of the National Center of Competence in Research (NCCR) "Materials' Revolution: Computational Design and Discovery of Novel Materials (MARVEL)" of the Swiss National Science Foundation. We used computational resources of CSCS, SCITAS, and CSEA-EPFL.

\section{References}

1. R. Jinnouchi, A.B. Anderson, Phys. Rev. B 77(24), 245417 (2008)

2. H.F. Wang, Z.P. Liu, J. Phys. Chem. C 113(40), 17502 (2009)

3. N. Bonnet, N. Marzari, Phys. Rev. Lett. 110(8), 086104 (2013)

4. S. Sakong, M. Naderian, K. Mathew, R.G. Hennig, A. Groß, J. Chem. Phys. 142(23), 234107 (2015)

5. V. Tripkovic, M.E. Björketun, E. Skúlason, J. Rossmeisl, Phys. Rev. B 84(11), 115452 (2011)

6. E. Skúlason, G.S. Karlberg, J. Rossmeisl, T. Bligaard, J. Greeley, H. Jónsson, J.K. Nørskov, Phys. Chem. Chem. Phys. 9(25), 3241 (2007)

7. S. Sakong, K. Forster-Tonigold, A. Groß, J. Chem. Phys. 144(19), 194701 (2016)

8. J. Le, M. Iannuzzi, A. Cuesta, J. Cheng, Phys. Rev. Lett. 119(1), 016801 (2017)

9. A.Y. Lozovoi, A. Alavi, J. Kohanoff, R.M. Lynden-Bell, J. Chem. Phys. 115(4), 1661 (2001)

10. N. Bonnet, T. Morishita, O. Sugino, M. Otani, Phys. Rev. Lett. 109(26), 266101 (2012)

11. M. Otani, O. Sugino, Phys. Rev. B 73(11), 115407 (2006)

12. M. Otani, I. Hamada, O. Sugino, Y. Morikawa, Y. Okamoto, T. Ikeshoji, Phys. Chem. Chem. Phys. 10(25), 3609 (2008)

13. M. Otani, I. Hamada, O. Sugino, Y. Morikawa, Y. Okamoto, T. Ikeshoji, J. Phys. Soc. Jpn. 77(2), $024802(2008)$

14. O. Sugino, I. Hamada, M. Otani, Y. Morikawa, T. Ikeshoji, Y. Okamoto, Surf. Sci. 601(22), $5237(2007)$

15. J. Cheng, M. Sprik, Phys. Chem. Chem. Phys. 14(32), 11245 (2012)

16. M.H. Hansen, C. Jin, K.S. Thygesen, J. Rossmeisl, J. Phys. Chem. C 120(25), 13485 (2016)

17. J. Rossmeisl, K. Chan, R. Ahmed, V. Tripković, M.E. Björketun, Phys. Chem. Chem. Phys. 15(25), 10321 (2013)

18. S. Nosé, Mol. Phys. 52, 255 (1984)

19. S. Nosé, J. Chem. Phys. 81(1), 511 (1984)

20. W.G. Hoover, Phys. Rev. A. 31(3), 1695 (1985)

21. P.E. Blöchl, M. Parrinello, Phys. Rev. B 45, 9413 (1992). DOI 10.1103/PhysRevB.45.9413. URL http://link.aps.org/doi/10.1103/PhysRevB.45.9413

22. H.C. Andersen, J. Chem. Phys. 72(4), 2384 (1980)

23. O.A. Vydrov, T. Van Voorhis, J. Chem. Phys. 133(24), 244103 (2010)

24. R. Sabatini, T. Gorni, S. de Gironcoli, Phys. Rev. B 87(4), 041108 (2013)

25. G. Miceli, S. de Gironcoli, A. Pasquarello, J. Chem. Phys. 142(3), 034501 (2015)

26. N. Troullier, J.L. Martins, Phys. Rev. B 43(3), 1993 (1991). DOI 10.1103/PhysRevB.43.1993

27. P. Giannozzi, S. Baroni, N. Bonini, M. Calandra, R. Car, C. Cavazzoni, D. Ceresoli, G.L. Chiarotti, M. Cococcioni, I. Dabo, et al., J. Phys. Condens. Matter 21(39), 395502 (2009)

28. F. Ambrosio, G. Miceli, A. Pasquarello, J. Chem. Phys. 143(24), 244508 (2015)

29. A. Bouzid, A. Pasquarello, J. Chem. Theory Comput. 13(4), 1769 (2017) 
30. J.F. Janak, Phys. Rev. B 18(12), 7165 (1978)

31. R.A. Marcus, J. Chem. Phys. 43(2), 679 (1965)

32. R.A. Marcus, J. Chem. Phys. 24(5), 966 (1956)

33. J. Cheng, X. Liu, J. VandeVondele, M. Sulpizi, M. Sprik, Acc. Chem. Res. 47(12), 3522 (2014)

34. J. Cheng, J. VandeVondele, Phys. Rev. Lett. 116(8), 086402 (2016)

35. A. Bouzid, A. Pasquarello, J. Phys. Chem. Lett. 9(8), 1880 (2018)

36. M. Todorova, J. Neugebauer, Phys. Rev. Appl. 1(1), 014001 (2014)

37. T. Ikeshoji, T. Uchida, M. Otani, M. Osawa, J. Electroanal. Chem. 800, 13 (2017)

38. T. Ikeshoji, M. Otani, Phys. Chem. Chem. Phys. 19(6), 4447 (2017)

39. M. Sprik, G. Ciccotti, J. Chem. Phys. 109(18), 7737 (1998)

40. G. Ciccotti, R. Kapral, E. Vanden-Eijnden, Chem. Phys. Chem. 6(9), 1809 (2005)

41. E. Gileadi, S. Argade, J.O. Bockris, J. Phys. Chem. 70(6), 2044 (1966)

42. R. Rizo, E. Sitta, E. Herrero, V. Climent, J.M. Feliu, Electrochim. Acta 162, 138 (2015)

43. C.G. Malmberg, A.A. Maryott, J. Res. Nat. Bureau Stand. 56, 1 (1956)

44. T. Pajkossy, D. Kolb, Electrochim. Acta 46(20), 3063 (2001)

45. T. Pajkossy, D. Kolb, Electrochem. Commun. 5(4), 283 (2003)

46. N. Garcia-Araez, V. Climent, E. Herrero, J.M. Feliu, J. Lipkowski, Electrochim. Acta 51(18), 3787 (2006)

47. D. Kolb, Surf. Sci. 500(1), 722 (2002)

48. T. Iwasita, X. Xia, J. Electroanal. Chem. 411(1-2), 95 (1996)

49. N. Garcia-Araez, V. Climent, J. Feliu, J. Phys. Chem. C 113(21), 9290 (2009)

50. M. Osawa, M. Tsushima, H. Mogami, G. Samjeske, A. Yamakata, J. Phys. Chem. C 112(11), $4248(2008)$

51. K.i. Ataka, T. Yotsuyanagi, M. Osawa, J. Phys. Chem. 100(25), 10664 (1996) 